\title{
Quais Deveriam Ser Os Rumos Dos Sistemas De Saúde Após A Pandemia De Covid-19?
}

César Monte Serrat Titton

\author{
Médico de Família e Comunidade. Professor do curso de Medicina da \\ Faculdade Evangélica Mackenzie do Paraná (FEMPAR). Mestrando em \\ Saúde da Família pela UFPR (PROFSAÚDE/FIOCRUZ). Ex-Secretário
} da Saúde de Curitiba (2015-2016).

email

“Em geral a saúde não é percebida, é um pouco como o ar que respiramos, de que apenas sentimos falta quando não o temos" (1). Talvez os sistemas de saúde sofram de uma invisibilidade relativa semelhante na maior parte do tempo - mas certamente ficam em evidência durante uma pandemia como a que estamos vivendo. Torna-se difícil não pensar na saúde e nos sistemas de saúde mas, se o foco de atenção ficar sequestrado apenas neste prolongado presente pandêmico, podem ser perdidas oportunidades de repensar tempestivamente os rumos dos sistemas de saúde para o período pós-pandemia.

Estender os olhares ao futuro agora pode, contudo, levar a planos estreitados pelas circunstâncias vigentes. Isso pode até ser vantajoso, por permitir foco e detalhamento nos discursos e no planejamento de ações para se preparar melhor para "as próximas pandemias" (2), mas não basta: um sistema de saúde não deve ser só um sistema de enfrentamento de pandemias - o ideal, aliás, seria manter o amplo espectro de ações dos sistemas de saúde mesmo durante uma pandemia. Apesar disto, temos assistido a diversos exemplos nos quais a ênfase do enfrentamento da pandemia tem sido dada apenas a certos componentes dos sistemas de saúde, como a atenção hospitalar (3-5) - o que amplia os riscos das sociedades viverem várias ondas de morbimortalidade além da diretamente associada com o efeito agudo da Covid-19 em si $(6,7)$.

Além de discutir os sistemas de saúde em geral, é interessante se debruçar sobre o caso aqui do Brasil, onde o Sistema Único de Saúde - SUS, que costumava sofrer não só de invisibilidade mas até mesmo de uma opinião pública negativa, passou a ser amplamente reconhecido na sociedade brasileira (8). Mais que isso: alguns, como Ferreira, Britto e Santos (9), chegam a entender até que "um efeito inesperado desta pandemia é o fortalecimento da ideia de que a atenção e o cuidado à saúde devem estar sob o olhar atento do Estado". Tal entendimento, porém, não é consenso - Guimarães (10) aponta, por exemplo, que diversos "amigos da hora" do SUS propõe na verdade formas de convergência público-privada.

A partir de tais reflexões, passamos agora para um panorama resumido dos rumos que vêm sendo apontados por pesquisadores para os sistemas de saúde em geral, com especial atenção para referências vinculadas ao SUS.

Sendo a pandemia de Covid-19 um desafio de saúde extraordinário em escala global, pode parecer que as demandas mais apontadas para os sistemas de saúde tenham que ser também extraordinárias e inovadoras. Impressiona, entretanto, como a maior parte das discussões a respeito tem tratado de transformações que já eram reconhecidas como necessárias antes da pandemia(11). Isto pode ser desalentador, por indicar oportunidades perdidas de estarmos em melhor situação agora, mas pode ser um ponto positivo por tratar de temas já conhecidos e já implantados em alguns sistemas de saúde, mesmo que nem sempre com força e alcance suficientes. Dentre estas tendências, são apontadas como mudanças necessárias aos sistemas de saúde pós-pandemia: 
1. Intensificar os cuidados de proximidade: cuidados de base territorial (extra-hospitalares(12)) centrados nas tecnologias leves capazes de produzir cuidados de ala complexidade que podem reconfigurar o modelo de atenção. Desenvolvidos na atenção primária à saúde - APS, na atenção domiciliar (seja da APS ou não), ou nos demais serviços de saúde de âmbito comunitário (como os de Saúde Mental)(4).

1.1. Desenvolver uma APS forte (13), tanto para enfrentar a pandemia quanto para enfrentar as necessidades de saúde pós-pandemia, por viabilizar mais acesso, cuidados continuados para pessoas em situação de sofrimento e/ou portadoras de condições crônicas, ações preventivas e educativas apropriadas culturalmente e vigilância em saúde integrada $(3,7,14)$; APS associada a participação local e voltada para reduzir desigualdades em saúde (15);

1.2. Transformar os sistemas de Saúde Mental, colocando no centro as iniquidades e as conexões da Saúde Mental tanto com os determinantes sociais de opressão econômica e racial quanto com a emergência ecológica e climática global, por meio de participação ativa e liderança colaborativa de todas as partes interessadas (incluindo comunidades, não-especialistas e não-clínicos) (16).

1.3. Adaptar os sistemas de saúde aos desafios trazidos pelo envelhecimento da população, especialmente ao acesso nos territórios de atenção ((17); integrar com os sistemas de saúde os serviços voltados ao cuidado de pessoas idosas (12) e com os serviços de assistência social (18).

2. Aprimorar a vigilância em saúde em todos os sistemas de saúde, com preparação continuada (19); melhorias na agilidade dos sistemas de vigilância em saúde (2); governança apropriada para efetivar ações de vigilância (20); organização dos serviços de assistência à saúde em todo o sistema conforme demandas de vigilância em saúde, como separar atendimento de pacientes infectados de outras demandas (12).
1. Combater a desigualdade de acesso a insumos de saúde (vacinas, exames, oxigênio) e enfrentar lacunas nas capacidades de prevenir, detectar e responder a epidemias $(2,11)$.

2. Melhorar a infraestrutura (capacidade organizacional para coleta e distribuição de informação) no sistema de saúde, integrando assistência, vigilância e gestão e ampliando a capacidade de resposta com transparência (21); tornar instantâneo o fluxo de informações de vigilância em saúde e facilitar integração de informações (18).

3. Reduzir os cuidados de baixo valor: aproveitar a disrupção da pandemia para sair da inércia, atentos também aos impactos positivos da redução e adiamento de serviços de saúde, pois estima-se que $10-20 \%$ dos custos em saúde envolvem serviços com maior chance de causar dano ou com remotas chances de benefício clínico (22).

4. Garantir melhor financiamento público de saúde continuado (23) (pelo menos 6\% do PIB, sendo ao menos $30 \%$ para APS, é o postulado pela OPAS(11)); previsão de incremento imediato de financiamento para enfrentar crises de saúde futuras (2).

5. Aumentar a força de trabalho pública em saúde (18); agilidade para redirecionamento dos profissionais de saúde (12); profissionais preparados para atuar em times multidisciplinares (15)e para exercer com criatividade e potência os cuidados de proximidade junto às comunidades (4).

Além disto, o contexto da pandemia de Covid-19 também criou novas tendências, algumas das quais já começaram a ser aplicadas, como as relacionadas com a revolução tecnológica da telessaúde:

I. Porta de entrada digital nos serviços de saúde em geral (12);

II. Telemedicina para ampliar acesso e monitoramento continuado na APS (24);

III. Ampliação do uso das tecnologias digitais para manejo clínico do paciente, diagnóstico por imagem, análise de riscos, rastreamento de casos, geolocalização, análise de dados e relatórios, desenvolvimento de ferramentas de autodiagnóstico e orientação à tomada de decisão, atendimento pré-clínico não presencial, agendamento online, teleconsultas, autoavaliação dos sintomas, etc. (25) 
Dentre as novas demandas colocadas para os sistemas de saúde, existem também algumas que ainda carecem de ações para sua implementação e/ou de discussões para estabelecer estratégias e consensos:

a) Autossuficiência em vacinas e insumos diagnósticos e terapêuticos (cadeia de insumos com maior resiliência) - pelo menos em nível regional, e com distribuição equitativa $(2,11,12)$;

b) Mais liderança e coordenação nos níveis internacional, regional e nacional (12), incluindo: uma Organização Mundial da Saúde mais independente, fortalecida e investida de autoridade; um novo tratado mundial contra pandemias; e um Conselho para Ameaças à Saúde Global (2);

c) Novo conceito para os sistemas de saúde, evitando o foco apenas no enfrentamento de emergências de saúde e tendo como objetivos centrais tanto a promoção à saúde quanto a segurança da saúde. Para tal, fomentar engajamento comunitário, abranger outras políticas públicas além da saúde, redirecionar investimentos e prevenir fragmentação do sistema e suas interfaces com outras políticas públicas, visando produzir sociedades mais saudáveis e resilientes (5)

Propostas como esta última, de reformulação conceitual dos sistemas de saúde apresentada pela Alliance for Health Policy and Systems Research (vinculada à Organização Mundial de Saúde (5) podem causar estranhamento em muitos brasileiros por conferir tom de inovação a um conjunto de conceitos muito semelhante aos princípios e diretrizes do SUS - ou até mais semelhantes a formulação de sistema originalmente proposta pelo movimento da Reforma Sanitária Brasileira dentro do "espírito de 1985", que não chegou a se refletir por completo nas normas do SUS desde sua criação $(10,20)$.

É claro, porém, que o conjunto de desafios enfrentados pelo SUS impediu a plena efetivação de tais princípios e diretrizes na realidade do sistema de saúde $(9,10,20,23)$ - e análises das razões disso podem amparar novas abordagens para concretizar mudanças no período pós-pandemia, talvez aproveitando a valorização e visibilidade do sistema no presente momento (8)

De todo modo, esta discrepância entre projetos e realidade dos sistemas de saúde não é exclusividade brasileira.
Mais ainda: mesmo países com sistemas de saúde bem avaliados, tanto em seus índices de segurança em saúde quanto nos de prontidão para enfrentamento de epidemias, acabaram tendo performance muito aquém da prevista nesta pandemia de Covid-19 (26,27). Assim, é importante lembrar que, apesar de muitas das demandas acima listadas se constituirem em defesas do que "deveria" ser feito nos sistemas de saúde, indicações de rumos como estas não são suficientes para que tais rumos se concretizem e melhorem os sistemas de saúde pós-pandemia. Como declarou Carissa Etienne, diretora da Organização Panamericana da Saúde, "Ouvimos muito diálogo sobre a resiliência e fortalecimento dos sistemas de Saúde, mas devemos transformar os discursos em ações" (11). Que cada um dos sobreviventes desta pandemia se esforce para contribuir com isto e participar da criação de sociedades mais saudáveis.

\section{REFERÊNCIAS}

1. Gérvas J, Fernández MP. São e Salvo - e livre de intervenções médicas desnecessárias. Porto Alegre: Artmed; 2016.

2. Independent Panel for Pandemic Preparedness and Response. COVID-19: Make it the Last Pandemic [Internet]. 2021. Available from: https://theindependentpanel.org/

3. Daumas RP, Azevedo e Silva G, Tasca R, da Costa Leite I, Brasil P, Greco DB, et al. O papel da atenção primária na rede de atenção à saúde no Brasil: limites e possibilidades no enfrentamento da COVID-19. Cad Saude Publica. 2020;36(6).

4. Seixas CT, Merhy EE, Feuerwerker LCM, Santo TB do E, Slomp Junior $H$, Cruz KT da. A crise como potência: os cuidados de proximidade e a epidemia pela Covid-19. Interface - Comun Saúde, Educ. 2021;25(suppl 1):1-15.

5. Shroff ZC, Marten R, Vega J, Peters DH, Patcharanarumol W, Ghaffar A. Time to reconceptualise health systems. Lancet [Internet]. 2021;397(10290):2145. Available from: http://dx.doi.org/10.1016/S0140-6736(21)01019-9

6. Babaian J. THE PANDEMIC'S 4TH WAVE [Internet]. April 7, 2020. 2020. Available from: https://hcldr.wordpress.com/2020/04/07/the-pande mics-4th-wave/

7. Douglas $M$, Katikireddi SV, Taulbut M, McKee M, McCartney G. Mitigating the wider health effects of covid-19 pandemic response. BMJ [Internet]. 2020;369(April):1-6. Available from: http://dx.doi.org/doi:10.1136/bmj.m1557 
8. Colucci C. A IMAGEM DO SUS DEPOIS DA PANDEMIA. In: Santos A de O (org. ., Lopes LT (org. ., editors. Reflexões E Futuro Volume 6 [Internet]. Brasília, DF: Conselho Nacional de Secretários de Saúde; 2021. p. 282-93. Available from: https://youtu.be/_NEJ6ZIN-cY

9. Ferreira PX, Britto NA, Santos A de O. O MUNDO QUE QUEREMOS: A PANDEMIA DE COVID-19 E REFLEXÕES SOBRE O FUTURO. In: Reflexões E Futuro Volume 6. Brasília, DF: Conselho Nacional de Secretários de Saúde; 2021. p. 102-113.

10. Guimarães R. O FUTURO DO SUS E A EMERGÊNCIA SANITÁRIA [Internet]. 28 de abril de 2021. 2021. Available from: https://www.cienciaesaudecoletiva.com.br/novidades/o-f uturo-do-sus-e-a-emergencia-sanitaria/146?id=146

11. Etienne C. Seminários Avançados de Saúde Global e Diplomacia em Saúde [Internet]. 2021. Available from: https://youtu.be/_NEJ6ZIN-cY

12. Britnell M. 10 ways that health systems are reacting and becoming more resilient [Internet]. KPMG. 2021. Available from: https://home.kpmg/xx/en/home/industries/healthcare/co vid-19-and-healthcare/ten-ways-health-systems-are-reacti ng-and-becoming-resilient.html

13. Organização Pan-Americana Saúde. 30 Anos De Que Para 2030? [Internet]. 2018. 222 p. Available from: http://iris.paho.org/xmlui/bitstream/handle/123456789/4 9663/9789275720448_por.pdf?sequence=1\&isAllowed=y

14. Favoreto CAO. ATENÇÃO PRIMÁRIA FORTE: ELEMENTO CENTRAL NO COMBATE À PANDEMIA DE COVID-19. In: Teixeira CP, Favoreto CAO, Santos DVD dos, Savassi LCM, Pinto MCRVGM de FASMMEB, editors. COVID-19 e Atenção Primária - As experiências nos territórios (Rede Profsaúde). Rio de Janeiro: ProfSaúde/FIOCRUZ; 2020. p. 14-9.

15. VEILLARD J, URIBE MV. Improving the frontline of health care services post COVID-19: A key agenda for resilient health systems [Internet]. World Bank Blogs. 2021. Available from: https://blogs.worldbank.org/latinamerica/improving-front line-health-care-services-post-covid-19-key-agenda-resilie nt-health

16. Belkin G, Appleton S, Langlois K. Reimagining mental health systems post COVID-19. Lancet Planet Heal [Internet]. 2021;5(4):e181-2. Available from: http://dx.doi.org/10.1016/S2542-5196(21)00037-1

17. Guimarães RB, da Costa NM, Nossa PN. Territorial and urban health: From pre-pandemic and pandemic challenges to post-pandemic responses. Saude e Soc. 2020;29(2):1-5.
18. Ferrinho P. Seminários Avançados de Saúde Global e Diplomacia em Saúde. [Internet]. 2021. Available from: https://youtu.be/_NEJ6ZIN-cY

19. Freitas CM de, Cunha RV. A VIGILÂNCIA EM SAÚDE DIANTE DA COVID-19 - DESAFIOS E LIÇÕES PARA O ENFRENTAMENTO DE EMERGÊNCIAS EM SAÚDE FUTURAS. In: Santos A de O (org. ., Lopes LT (org. ., editors. Reflexões E Futuro Volume 6. Brasília, DF: Conselho Nacional de Secretários de Saúde; 2020. p. 152-69.

20. Lopes-júnior LC, Araújo Lacerda A, Fonseca Venâncio $F$, Peisino Buleriano L, Baltar Sobreira L. Vigilância em Saúde na pandemia de COVID-19 e os desafios do SUS na atualidade. Saúde Coletiva (Barueri). 2021;11(64):5714-27.

21. Ferré F. INFOESTRUTURA PARA APOIO À DECISÃO ESTRATÉGICA NO SUS. In: Santos A de O (org. ., Lopes LT (org. ., editors. Reflexões E Futuro Volume 6. Brasília, DF: Conselho Nacional de Secretários de Saúde; 2020. p. 114-35.

22. Sorenson $C$, Japinga $M$, Crook $H$, Mcclellan $M$. Building a better health care system post-covid-19: steps for reducing low-value and wasteful care. NEJM Catal [Internet]. 2020;1-10. Available from: https://wahealthalliance.org/wp-content/uploads/2013/1 $1 /$ Choos-

23. Servo LMS, Santos MAB dos, Vieira FS, Benevides RP de S e. Financiamento do SUS e Covid-19: histórico, participações federativas e respostas à pandemia. SciELO Prepr [Internet]. 2021; Available from: https://doi.org/10.1590/SciELOPreprints.1530

24. Pinto MEB. TELEMEDICINA: FERRAMENTA PARA UM NOVO PARADIGMA NA APS. In: Teixeira CP, Favoreto CAO, Santos DVD dos, Savassi LCM, Pinto MCRVGM de FASMMEB, editors. COVID-19 e Atenção Primária - As experiências nos territórios (Rede Profsaúde). Rio de Janeiro: ProfSaúde/FIOCRUZ; 2020. p. 114-7.

25. Celuppi IC, dos Santos Lima G, Rossi E, Wazlawick RS, Dalmarco EM. Uma análise sobre o desenvolvimento de tecnologias digitais em saúde para o enfrentamento da COVID-19 no Brasil e no mundo. Cad Saude Publica. 2021;37(3).

26. Baum F, Freeman T, Musolino C, Abramovitz M, De Ceukelaire W, Flavel J, et al. Explaining covid-19 performance: What factors might predict national responses? BMJ. 2021;372:4-7.

27. Medici AC. Covid-19 no Brasil: Razões da Baixa Performance na Gestão da Pandemia [Internet]. Blog Monitor de Saúde. 2021. Available from: https://monitordesaude.blogspot.com/2021/02/covid-19no-brasil-razoes-da-baixa.html 